\title{
Aggregate Operation Movement: A Min-Cut Approach to Global Code Motion
}

\author{
Raymond Lo, Sun Chan, Jim Dehnert, Ross Towle \\ Silicon Graphics Computer Systems \\ P.O.Box 7311, Mountain View, CA 94039-7311
}

\begin{abstract}
This paper describes a novel alternative to trace scheduling and other global scheduling techniques that attempt to boost instruction level parallelism by moving operations beyond basic block boundaries. We quantify the relative benefits of moving operations from one basic block to another with respect to critical path length, register pressure, and avoiding interlocks from long-latency operations. The benefits are encoded as flow capacities in a network, and a mincut algorithm is used to select the set of operations to move. Unlike other approaches, our method is applied before register allocation and scheduling. Our experiments on a superscalar processor show that significant speedup can be obtained for both integer and floating-point benchmarks using this method, even in the presence of an excellent software pipeliner.

Keywords: global scheduling, global code motion, software pipelining, superscalar, network flow, minimum cut.
\end{abstract}

\subsection{Introduction}

Most modern processors benefit from the rearrangement of operations to avoid interlocks between instructions. In earlier processors, applying such scheduling to one single basic block (BB) at a time is adequate. But the instruction level parallelism (ILP) available within individual $\mathrm{BBs}$ is not sufficient to fully exploit the multiple resources present in today's superscalar and VLIW architectures. It is essential to move operations past BB boundaries to achieve higher ILP. This activity is called global scheduling, since most such algorithms incorporate instruction scheduling. A good overview of ILP issues may be found in [RaFi93].

Much work has been done on global scheduling for inner loop bodies, with software pipelining in particular being an effective solution ([Char+81], [RaG181], [Touzeau84], [DeTo93], and [RGSL96]). But global scheduling is also important outside loops, and for dealing with those inner loops which cannot be pipelined.

We present a new approach to global scheduling, which we call Aggregate Operation Movement (AOM), reflecting the unique characteristic that it decides on a partition of instructions between a pair of basic blocks in the aggregate, rather than operation by operation. AOM provides a framework within which one can apply a variety of heuristics; we describe those we have used and preliminary results obtained.

In the remainder of this section, we summarize AOM and compare it to previous work. In Section 2, we discuss our implementation context - the motions attempted, and how they are constrained. In Section 3, we discuss our AOM implementation. In Section 4 , we provide a preliminary performance evaluation.

\subsection{Aggregate Operation Movement}

AOM takes a strategic view of the global scheduling task, with the basic objective of partitioning critical path length between the $\mathrm{BBs}$ in a flowgraph, leaving the actual instruction scheduling to the BB scheduler. The key idea is to construct a network flow 
model of a pair of BBs, based on its data dependency graph, and use a min-cut algorithm to partition the instructions between the pair of BBs. The key contributions of this approach are:

- AOM separates the operation movement decisions from instruction scheduling. It is thus a separate, self-contained transformation phase, with significant freedom as to where it may be applied within the compiler. It may use as much or as little global information in its decisions as is available and cost-effective, including execution frequencies from either profile feedback or static estimates.

- AOM considers all legally movable operations in a BB for code motion at once, and moves them as an aggregate, thus avoiding the overhead of incremental update. This allows us to iterate over the flow graph until convergence at a modest compile time cost (approximately $10 \%-20 \%$ of the optimization and code generation time).

- AOM is based on a network flow model of the code motion problem, allowing it to use traditional min-cut algorithms (e.g., [FoFu62]) to choose the operations to move. Within this framework, a variety of heuristics can be used in building the network flow graph. It is similar to the approach taken in [Stone77][Lo88] to solve multi-processor scheduling problems.

- We have implemented a unified cost model of operation movement, combing critical path length, register pressure, and latency subsumption goals in a single flow capacity function. This model is flexible in that the weights of its components can be adjusted for different situations, and additional cost components could be handled in the same manner.

- We use a simple model of safety which gives the user flexibility in trading off better optimization against better exception detection.

AOM is applied to pairs of (not always adjacent) BBs, iterating over the flow graph to produce extended motion. AOM can achieve effects similar to software pipelining (SWP), but a traditional SWP algorithm can do a better job of scheduling tight loops by modeling the hardware very closely. However, the loops most amenable to SWP usually do not have a large number of branches, and must be innermost in a loop nest. In our implementation, AOM is intended as a supplement to SWP, handling non-innermost loops and loops with complicated control structures as well as acyclic code.

\subsection{Comparison to Previous Work}

There have been several important approaches to global scheduling reported.

Trace scheduling ([LFKL+93], [Ellis86], [Fisher81]) reduces global scheduling to a local scheduling problem by scheduling a trace (an acyclic path) in the flowgraph and allowing operations to move past branches or labels within the trace, inserting fixup code to correct for changes due to such movement past the branches. Trace scheduling handles loops by breaking traces at back arcs, using unrolling to mitigate the resultant inability to move code past those arcs.

Trace scheduling optimizes the first traces scheduled at the cost of fixup code on the side traces, even if they have equal execution frequency, making it sensitive to the selection of traces. Superblock scheduling ([HMCC+93]) mitigates this problem somewhat by replicating parts of the flowgraph, reducing the number of transitions between traces. AOM instead treats all paths the same by looking at all nearby BB pairs. It dis- 
criminates only in that the first pairs considered get first claim on available resources. As a result, it is much less dependent on execution profiling or frequency estimates. It also avoids the extensive unrolling used by trace scheduling, handling back arcs as effectively as forward arcs.

Percolation scheduling [Nicolau86] is based on four primitive transformations for moving instructions. It attempts unbiased treatment of conditional branches, replicating instructions as they move up past joins and recombining identical instructions moved up past a conditional branch. Because it ignores resource requirements, operations that are executed with small probability consume resources that could otherwise perform useful work. Enhanced percolation scheduling ([EbNi89], [NaEb93]) addresses this problem by delaying movement of operations until scheduling time. This postpones the movement decisions until actual machine resource requirements are known, restraining movement of operations that would exceed resource availability.

Global instruction scheduling [BeRo91] permits movement of operations beyond basic block boundaries within the scope of an enclosing loop, either to blocks with equivalent control dependence or speculatively above a conditional branch. It uses an enhanced block scheduler that improves the ILP of a basic block by considering operations from equivalent or successor blocks. The initial implementation avoids code duplication, handling loops by copying the first basic block to the end of the loop.

Percolation scheduling moves operations upward even if doing so is detrimental. Enhanced percolation scheduling suppresses motion which exceeds resource availability, but will still do useless motion which increases resource requirements. Global instruction scheduling similarly constrains motion based on available resources. Both of these approaches will greedily move operations until resources (e.g. registers) are used up, even if the movement does not help the schedule. They also stop movement when the resource limit is reached, even if the scheduling benefit would exceed the resource overflow cost (e.g. register spill/restore).

All of these techniques move a single operation at a time, either explicitly or implicitly by scheduling it outside its original BB. This limits their ability to make truly global tradeoffs in deciding where to place operations. It increases the cost of compiler decision-making by making a distinct choice for each operation, and often requires update of dependency information after each decision.

Unlike any of the other approaches described, AOM treats a source and target BB symmetrically, balancing the critical paths and resource usage between them. AOM can perform movements that worsen the target BB's schedule if they improve the source BB's schedule more. It will also leave operations in place even though unused resources are available in the target $\mathrm{BB}$ if the movement does not benefit the overall schedule. This limits useless code expansion. Furthermore, AOM handles loops well without special casing, by moving operations across the back-arc to previous iterations, producing an effect resembling software pipelining ([Jain91], [NaEb93]).

\subsection{A Context for AOM Implementation}

Our implementation of AOM is part of the MIPSpro compiler for the R8000 processor. It is applied to an intermediate representation of the program that is essentially at the machine operation level, with full target resource and latency information available. It 
comes after traditional global optimization and software pipelining, so it is able to avoid pipelined loops and free to improve those loops which cannot be pipelined. It is followed by instruction scheduling, local and global register allocation.

\subsection{Types of Code Motion}

In this paper, we only consider upward code motions, i.e., moving code to a preceding BB. Based on limited experience, downward code motion only gives marginal benefit. We distinguish four types of code motion: equivalent code motion, speculative code motion, code duplication, and circular code motion. The first may move code across multiple BBs, while the others only move code to adjacent BBs. The following descriptions is with reference to the flowgraph in Figure 1.

- Equivalent Code Motion: Two BBs are control equivalent iff the first BB dominates the second $\mathrm{BB}$, the second postdominates the first, and they are at the same loop-nest level. Code moved from $B B_{b}$ to $B B_{a}$ is called upward equivalent code motion. Exceptions are not an issue for such motion, as long as data dependency constraints are observed, because only the order of execution is changed. We perform this motion if $B B_{a}$ and $B B_{b}$ are an immediate dominator-postdominator pair.

- Speculative Code Motion: Moving an operation from $B B_{c}$ to $B B_{a}$ is speculative if $B B_{c}$ does not postdominate $B B_{a}$. The operation moved may be executed unnecessarily if $B B_{c}$ is not subsequently executed, and we must therefore consider the possibility of introducing exceptions. Nevertheless, we consider this motion if $B B_{a}$ is the only predecessor of $B B_{c}$.

- Code Duplication: Moving an operation from $B B_{b}$ to $B B_{c}$ requires its duplication in $B B_{d}$ if $B B_{c}$ does not dominate $B B_{b}$. This motion is applied when $B B_{b}$ is the only successor of its predecessors, and there is no path connecting $B B_{c}$ and $B B_{d}$ without passing through $B B_{b}$.

- Circular Code Motion: This involves moving operations from the top of a loop to its bottom (sometimes called bottom loading). Viewing back arcs like any other arcs in the flow graph, its legality condition is the same as for speculative code motion (because of other successors of $B B_{b}$ ), but its transformation is the same as code duplication (because of other predecessors of $B B_{a}$ ). We choose to differentiate this kind of movement from other code motions because of the loop context.

We apply AOM to pairs of basic blocks which are either adjacent in the flowgraph or are equivalent, covering the cases described above. We control this process as follows:

1. The program flow graph is topologically sorted (ignoring back arcs). AOM is applied bottom up, so operations move up as much as possible in a single traversal of the flow graph.

2. The algorithm is applied to the flow graph repeatedly until no further movement occurs. Our experiments show that AOM seldom requires more than six iterations. To prevent excessive compilation time, we limit the iteration count to six.

3. In each iteration, the BB pairs and thus types of motion are considered in order of their potential benefits relating to their costs. Circular code

Figure 1. Types of code motion

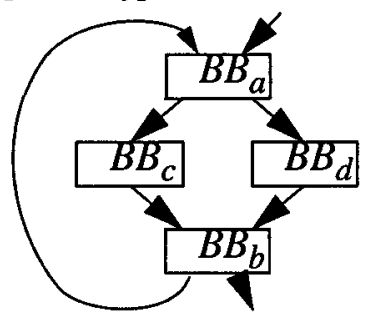


motion is tried first because BBs in a loop have higher execution counts. Equivalent code motion does not insert extra operations, so it is performed next. Code duplication inserts extra copies of operations, but does not increase any path length, so it is applied third. Speculative code motion is performed last since it may increase path lengths if branch prediction is poor. Among speculative motions, we apply static heuristics [BaLa93] to select the BB pairs with highest edge probability.

4. To avoid useless applications of the min-cut algorithm, we keep track of the motion attempted. We do not consider a pair of BBs if the same pair has been processed in a previous iteration and the BBs haven't been changed since then. As the algorithm iterates, each pass will require less time.

\subsection{Constraints on Motion}

Our algorithm requires identifying the maximal set of operations that may be moved from a given source $\mathrm{BB}$ to the target $\mathrm{BB}$ being considered, without changing the semantics of the program. Each of the operations in this maximal movable set $\left(M_{s}\right)$ must satisfy operation constraints, global constraints, and local constraints.

- Operation constraints identify operations that may not be moved. In this implementation, we preserve the flowgraph, so we do not consider moving calls and branches. Operations that have non-maskable side effects (e.g. stores) may not be moved speculatively. Many operations are unsafe to move speculatively because they might cause unrecoverable faults. However, we have taken an aggressive approach to moving operations which may fault, described below.

- Global constraints are due to the dependence on operations in basic blocks other than the source and target. An operation cannot be moved from $B B_{b}$ to $B B_{a}$ if its operands are defined, or its result is defined or used, on that path. An operation cannot be moved speculatively from $B B_{c}$ to $B B_{a}$ if its result has an exposed use in another successor of $B B_{a}$. An operation cannot be moved circularly from $B B_{a}$ to $B B_{b}$ if its result has an exposed use in any successor of $B B_{b}$.

- Local constraints are due to dependence on operations in the source or target BB. An operation is not movable if any operation that it depends on is not movable. A movable operation may be moved only if all of the operations upon which it depends are moved.

As noted above, speculative motion of many operations is unsafe in general. The most important examples are memory loads, which may cause addressing faults, and floating point operations. We use a simple model of exceptions which allows us to be aggressive about motion without ignoring safety considerations.

We first classify operations according to the nature of exceptions they may cause. Loads of named scalars can be moved because their addresses are always valid, as can some array element loads if the indices are known to be in bounds, but most other loads may cause addressing faults, e.g. due to invalid pointers. Floating point operations are also classified according to the exceptions they may cause - we treat divideby-zero as most severe, then overflow and invalid operands.

By default, we do not allow motion of operations which may cause exceptions that are traditionally enabled, i.e., memory, divide-by-zero, overflow, and invalid operands. 
However, we provide user options to suppress certain exceptions. If used, these options cause the runtime initializer to disable the exceptions, and allow the compiler to move operations that may cause them.

\subsection{The Aggregate Operation Movement Algorithm}

The AOM algorithm identifies the best set of operations to move from the source BB $\left(B B_{s}\right)$ to the target $\mathrm{BB}\left(B B_{t}\right)$. It first identifies the maximal set of operations that can be safely moved to the target $\mathrm{BB}$. The movable set is then transformed into a flow network [FoFu62] [EdKa72] in which capacities are assigned according to the cost of moving that operation. Finally, the mincut algorithm suggested by Edmond and Karp [EdKa72] is applied to determine the minimum cost set of operations to be moved, and they are all moved in one step.

\subsection{Motivation}

Moving all operations that are legal to move is often not profitable, for instance because it increases resource usage, or because it results in very unbalanced latency among the paths in the target BB. An effective heuristic must attempt to optimize the subset of the movable operations actually moved. However, examining one operation at a time for profitability is too limiting to produce optimal solutions unless an exhaustive algorithm is used. For example, a bushy expression may exceed the algorithm's register usage constraints at some intermediate stage, but moving the ntire expression would have no net effect on register usage at the BB boundary.

Given a $B B_{s}$, consider the DAG whose nodes are the movable operations and whose edges represent dependencies due to local constraints. The problem of finding a legal subset to move is equivalent to finding a bi-partition or a cut on the DAG such that all local constraints are satisfied. After assigning a movement cost to each operation in $M_{s}$, the problem of finding the optimal movement subset (relative to this heuristic cost function) can be formulated as a network flow problem and solved using a min-cut algorithm. The min-cut algorithm not only minimizes the cost function, but also guarantees the legality of the subset chosen, in particular moving operations only if their dataflow predecessors are all moved.

\subsection{Constructing the Flow Network}

Our transformation from the DAG of $M_{s}$ to a flow network ensures that each operation in $M_{s}$ has an arc corresponding to it, with a capacity representing its movement cost. We transform the dependency graph as follows.

1. Add a source and a sink node. Add arcs (called source-arcs) to connect the source node to the nodes in the DAG that have no predecessors. Add arcs (called sinkarcs) to connect the nodes in the DAG that have no successors to the sink node.

2. For each node, say $x$, in the DAG, split $x$ into two nodes: $x^{\prime}$ and $x^{\prime \prime}$, and add an arc (called an operation-arc) to connect $x^{\prime}$ and $x^{\prime \prime}$. Edges into $x$ become edges into $x^{\prime}$, and edges out of $x$ become edges out of $x$ ". This yields a 1-1 correspondence between operations and operation-arcs.

3. Assign infinite capacity to the arcs that are in the original DAG (called dependence-arcs) and the sink-arcs, ensuring that they will never be cut by the min-cut algorithm. The operation-arcs and source-arcs have their capacity determined by the cost functions described below. 
A min-cut algorithm ([EdKa72], [FoFu62]) identifies a partition of the flow network into two sections, one containing the source and the other containing the sink, by cutting arcs with minimum total capacity. Given such a partition, AOM moves the operations which end up in the source node's partition, including those operations $x$ corresponding to operation-arcs $\left(x^{\prime}, x^{\prime \prime}\right)$ that are cut, and all of their predecessors. If the cut passes through only source-arcs, no operations are moved.

We apply the min-cut algorithm to the restriction of the dependency graph to $M_{s}$. Since the moveable set is usually not very large, and its dependency graph is normally simple (i.e. acyclic, $\mathrm{O}(\mathrm{n})$ edges), our experience is that the min-cut algorithm consumes only a small part of the compile time.

\subsection{Cost/Benefit Quantification}

Accurately quantifying a flow capacity, i.e., the cost or benefit of moving an operation, directly affects the effectiveness of the min-cut approach. We use heuristics which consider three factors in the cost of moving an operation: critical path length, global register pressure, and subsumed latency. We determine flow capacities based on each of these factors as if it were the only cost component. We then produce summary flow capacity as the weighted sum of the three components. The weighting can be varied depending on the situation. We use different weights for the different classes of code motion, for example giving register pressure less weight for movement between adjacent blocks than for motion across intermediate blocks. A detailed example is in the Appendix.

Our min-cut formulation requires that flow capacities (i.e., costs) be assigned on the edges of the dependency graph of $M_{s}$. However, the computation of cost/benefit is based on the combined dependency graph of $B B_{t}$ and $B B_{s}$, including non-movable operations.

\subsubsection{Critical Path Balancing}

Given a pair of BBs, the objective of $A O M$ is to minimize the total cycles needed to execute the two BBs. Rather than resort to scheduling, AOM attempts to minimize the total critical path length of two BBs because the total length represents a lower bound on the cycles needed.

We merge the dependency graphs of $B B_{s}$ and $B B_{t}$, and treat all non-movable operations in the source as dependent on all target operations by adding dependence arcs with latency 1 from the stop node of $B B_{t}$ to the non-movable operations. Then analyze critical paths through the combined graph. We can thereby identify those operations of $B B_{s}$ (which includes the non-movable subset $N_{s}$ of $B B_{s}$ ) that must start after the end of the longest critical path through $B B_{t}$. The operations which are potentially beneficial to move are those movable operations of $B B_{s}$ that can start earlier than $N_{s}$. We assign zero cost to a set of arcs which yields such a partition, and higher costs when operations are further from that boundary.

More precisely, each operation in $B B_{s}$ and $B B_{t}$ is assigned a pair (estart, lstart) where estart is the earliest start cycle of an operation based on the latency of all paths to it from the start node. The length of a $\mathrm{BB}, \operatorname{len}(B B)$, is the value of estart of the stop node. Given lstart=estart for the stop node, lstart for the other operations is the latest 
start cycle possible based on the latency of all paths from it to the stop node. Note that the estart of a non-movable operation $B B_{s}$ must be greater than the estart of any operation of $B B_{t}$. estart and lstart are computed as follows:

estart $(o p)=\max ($ estart $($ pred $)+\operatorname{latency}($ pred,$o p))$, for each predecessor of $o p$.

lstart $(o p)=\min ($ lstart $($ succ $)-$ latency $($ op, succ $))$, for each successor of op.

Let $E_{t}=\operatorname{len}\left(B B_{t}\right)+1$. For each op, we compute the earliest start time (estart) and latest finish time (lfinish) of its associated arcs in the network as follows.

estart $(\operatorname{arc})=\operatorname{estart}(o p)-E_{t}$, if $\operatorname{arc}$ is a source-arc ending at $o p$.

lfinish $(\operatorname{arc})=l$ start $(o p)-E_{t}$, if arc is a source-arc ending at op.

estart $(\operatorname{arc})=e s t a r t(o p)-E_{t}$, if $\operatorname{arc}$ is an operation-arc starting at $o p$.

lfinish $(\operatorname{arc})=l$ start $(o p)+$ latency $(o p)-E_{t}$, if arc is an operation-arc ending at $o p$.

Based on these calculations, the capacity component based on critical path length $\left(C_{\text {path }}\right)$ is a function of estart(arc) and lfinish(arc) as defined in Table 1. Note that $C_{\text {path }}(\operatorname{arc})>=0$ for all arcs.

TABLE 1. Critical Path Cost Assignment.

\begin{tabular}{|c|c|c|c|}
\hline$C_{\text {path }}$ (arc) & estart(arc) & lfinish(arc) & Comment \\
\hline- lfinish(arc) & $<0$ & $<0$ & profitable to move \\
\hline 0 & $<0$ & $>=0$ & don't care \\
\hline estart(arc) & $>=0$ & $>=0$ & not profitable to move \\
\hline
\end{tabular}

If the critical path were the only component of the cost function, the resultant cut would always be through network nodes with capacity 0 , minimizing the total length of $B B_{s}$ and $B B_{t}$. A cut at arcs with non-zero capacity involves moving or failing to move the operation at the cost of increasing total path length.

\subsubsection{Latency Subsumption}

Given a pair of equivalent BBs separated by the branches of an IF, the cycles needed to transit from the exit of $B B_{t}$ to the entry of $B B_{s}$ is non-zero. We can take advantage of the long transit time by starting a long-latency operation in $B B_{t}$ instead of waiting until $B B_{s}$ starts executing. The result of the long-latency operation may then be ready when needed by its successor operations in $B B_{s}$. We call this optimization latency subsumption because the latency of the operation is subsumed by the cycles needed to execute the path from $B B_{t}$ to $B B_{s}$. Simply putting the longest latency operation of the movable set in $B B_{t}$ and the operations using its result in $B B_{s}$ works very well.

The operations which benefit most from this consideration on our target machine are generally those which tie up scarce resources, i.e. using execution units in an unpipelined manner, so that the choice of one or two for movement will saturate the available resources. For equivalent $\mathrm{BBs}$ that are close to each other or for adjacent $\mathrm{BBs}$ that can only subsume a few cycles (because branching from $B B_{t}$ to $B B_{s}$ takes a few cycles), the number of cycles that can be subsumed may be small compared to the latency of these operations, so that moving an operation with shorter latency may save just as much. Therefore, the decision to move an operation is a function of the operation's latency and the maximum latency that can be subsumed between $B B_{t}$ and $B B_{s}$.

We define the distance $(D)$ between $B B_{s}$ and $B B_{t}$ to be the number of cycles needed to go from $B B_{t}$ to $B B_{s}$. It is the maximum number of cycles required by some path 
from $B B_{t}$ to $B B_{s^{.}} D$ is estimated to be the maximum critical path length from $B B_{s}$ to $B B_{t}$ if $B B_{s}$ and $B B_{t}$ are equivalent, or as 0 if $B B_{t}$ falls through to $B B_{s}$. If $B B_{t}$ is a branch target of $B B_{s}, D$ is estimated to be the branch latency.

Suppose the longest latency in $B B_{s}$ is $L_{\max }$. The maximum benefit (subsumable latency) of moving an operation from $B B_{s}$ to $B B_{t}$ is $\min \left(L_{m a x} D\right)$ cycles. The capacity component $C_{\text {latency }}$ for an operation with latency $L$ is as follows:

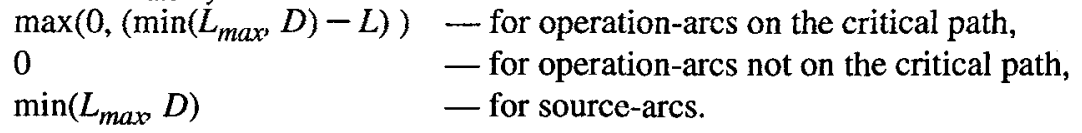

Intuitively, when subsumption of latency is the only component of the cost function, the result cut should always be along the arcs with capacity 0 . This moves the operation with longest latency. The capacity of an operation is the number of cycles that would be wasted if that operation were the last one moved instead of an operation with latency $L_{\max }$.

\subsubsection{Register Pressure}

The aggregate motion algorithm partitions the movable set into two halves by applying a cut across the dependency graph. Cutting an operation-arc represents moving an operations with successors that will remain in $B B_{s}$, so in general a register must be allocated to its result across the boundary between $B B_{t}$ and $B B_{s}$. If the number of registers required is less than the number of registers available for assignment, the partition chosen is not significant. Otherwise, because registers must be spilled to satisfy the increased demand and spilling is not free, we should minimize the register requirement when partitioning the dependency graph.

An operation frees a register if an operand is at the end of its live-range. An operation requires an extra register if defines a result. Moving an operation reduces register pressure if its net effect is to free registers. By assigning the number of values defined by an operation to be the capacity of an arc, the min-cut algorithm determines the partition that requires the minimum number of registers to hold the intermediate results between the source and target BBs.

$C_{r e g}(\operatorname{arc})=$ number of definitions for operation-arcs.

$C_{\text {reg }}($ arc $)=$ number of operands for source-arcs.

$C_{r e g}$ determines the capacities of the source-arcs and operation-arcs in the flow network. Recall that other arcs have infinite capacities, therefore, a min-cut only cuts through source-arcs and operation-arcs. Since each definition (register usage) has a capacity of one, the capacity of a cut is the total number of values (i.e., the number of registers needed to hold the temporary values) that are live from $B B_{t}$ to $B B_{s}$ if we move the operations above the cut from $B B_{s}$ to $B B_{t}$. If the cut is of minimum capacity, the cut determines the smallest amount of registers needed.

\subsubsection{Combining the Cost Components}

Each of the heuristics described above yields a cost function that could independently produce a partition of the movable set. By using a weighted sum in our cost quantification model, we are able to unify these heuristics and optimize the partition relative to their combination.

The fundamental concept in the quantification model is that each cut of an edge in 
the movable set (i.e., each movement) is associated with a cost. In this model, heuristics are no longer used to identify useful motions - they are used to identify the costs and benefits which determine whether motions are useful. The min-cut algorithm is then used to determine the optimal solution with respect to the heuristic cost model. We can model tradeoffs between two or more cost heuristics by a weighted sum of the associated flow capacities. The result of applying the min-cut algorithm is a partition that minimizes this weighted sum.

We believe that a global policy should be sensitive to program context. By allowing different weights depending on program context, a cost function is well suited for this purpose. Our implemented cost function, computed for each source-arc and operation-arc, is the weighted average of the critical path, latency, and register cost components. The first two are measured in cycles and are assigned the same weight $W_{c y c l e}$. The weight $W_{r e g}$ applied to register cost depends on the type of code motion and the global register pressure. We have $C_{\text {total }}=W_{\text {cycle }} *\left(C_{\text {path }}+C_{\text {latency }}\right)+W_{\text {reg }} * C_{\text {reg. }}$ The weighting $W_{\text {cycle }}$ for $C_{\text {path }}$ and $C_{\text {latency }}$ is 1 , if arc is a source arc. It is sum( $W_{\text {cycle }}$ (predarc) / out-degree (predarc)), for all preceding arcs predarc of arc, if arc is not a source arc.

Observe that if all arcs had $W_{\text {cycle }}$ defined above as their capacity, the maximum capacity of the flow network would be bounded by the number of source-arcs. Any cut would yield the same maximum capacity using this weighting. This weight assignment ensures that there is no inherent bias toward any specific cut.

For adjacent code motions, $W_{\text {reg }}$ is 0 , assuming live registers are no worse across a simple BB boundary than within a BB. For equivalent code motion, which moves operations across intervening BBs, we must account for the effect of global register pressure. When register pressure is high, moving an operation that consumes more registers causes spill, and the movement cost is the spill cost (one load-operation plus one store-operation). When the register pressure is low, moving that operation introduces no overhead. Hence, we vary the weight of register pressure component of the cost function based on the global register pressure. $W_{\text {reg }}$ for equivalent code motion is defined in Table 2, where availreg is the number of registers available for global register allocation, and needreg is the number of registers needed to hold global values and for local scheduling in the region between $B B_{s}$ and $B B_{t}$.

\subsection{Performance Results}

We have done a limited evaluation of the performance improvement due to AOM using the SPEC 92 benchmark suite. The benchmarks were run with full optimization (including software pipelining), with and without AOM, on a $75 \mathrm{MHz}$ MIPS R8000 processor (also known as TFP) [Hsu94]. The MIPS R8000 processor is a 4-issue

TABLE 2. Register Pressure Cost Assignment.

\begin{tabular}{|c|c|c|}
\hline Needreg & Register Pressure & $\mathbf{W}_{\text {reg }}$ \\
\hline$<$ availreg/2 & low & 0 \\
\hline $\begin{array}{c}\text { between availreg/2 } \\
\text { and availreg }\end{array}$ & medium & $\begin{array}{c}\text { spill_cost } \\
\text { (1-needreg/availreg })\end{array}$ \\
\hline$>$ availreg & high & spill_cost \\
\hline
\end{tabular}


superscalar RISC processor, implementing the MIPS IV instruction set. Its multi-chip implementation consists of two arithmetic logic units (ALU), two floating point units, and two load/store units. The design provides in-order dispatch of up to four instructions each cycle, making the R8000 a good candidate for global scheduling. Tables 3 and 4 list the user+system times (with and without AOM) measured in seconds, and the speedup obtained with AOM. With this approach, AOM achieves an average runtime improvement of $12 \%$ for SPEC92int and 7.3\% for SPEC92fp benchmarks, with a compile-time overhead of $10-20 \%$. The significant improvements are due to the AOM optimizations on loops that cannot be software-pipelined. Those exhibiting moderate to slight improvements are mostly due to the AOM's effect on the acyclic code.

\subsection{Future Work}

This paper has presented a set of cost functions to optimize critical path, register pressure, and latency subsumption. These functions are not the only way to exploit AOM. Using a different set of functions, it is possible to schedule for a different architecture or to implement a different optimization. For instance, the critical path information can be augmented with critical resources information if the earliest start time of an operation is defined to be later than the time for all its predecessors to release the required functional units. The increase/decrease of the number of live values needing transmission over the BB boundary can be more accurately determined by taking operands into account, using the following cost function.

number of definitions - number of operands unused later + number of operands

In our implementation, two BBs are considered at a time. By applying generalizations discussed in [Stone77] [Lo88], better code motion decisions can be made by considering all predecessors of a BB or all successors of it at the same time.

\subsection{Conclusions}

AOM differs from existing global scheduling techniques in that legality checking, the cost model, operation selection, and actual movements are independent, providing a

TABLE 3.SPEC92int Improvement

\begin{tabular}{|c|c|c|c|}
\hline SPEC_int & $\begin{array}{r}\text { AOM } \\
\text { Enabled } \\
\text { (in sec) }\end{array}$ & $\begin{array}{r}\text { AOM } \\
\text { Disabled } \\
\text { (in sec) }\end{array}$ & $\begin{array}{r}\text { Speedup } \\
\text { (in \%) }\end{array}$ \\
\hline 008.espresso & 27.55 & 31.75 & 15.2 \\
\hline $022 . l i$ & 68.45 & 81.38 & 18.9 \\
\hline 023. eqntott & 6.90 & 8.55 & 23.9 \\
\hline 026.compress & 34.97 & 36.90 & 5.5 \\
\hline 072.sc & 26.00 & 26.60 & 2.3 \\
\hline 085.gcc & 63.64 & 67.90 & 6.7 \\
\hline
\end{tabular}

SPEC92int improved $12 \%$ on average. SPEC92fp improved $7.3 \%$ on average.
TABLE 4.SPEC92fp Improvement

\begin{tabular}{|c|c|c|c|}
\hline SPEC_fp & $\begin{array}{r}\text { AOM } \\
\text { Enabled } \\
\text { (in sec) }\end{array}$ & $\begin{array}{r}\text { AOM } \\
\text { Disabled } \\
\text { (in sec) }\end{array}$ & $\begin{array}{r}\text { Speedup } \\
\text { (in \%) }\end{array}$ \\
\hline 013.spice2g6 & 289.32 & 346.87 & 19.9 \\
\hline 015.doduc & 11.74 & 13.98 & 19.1 \\
\hline 034.mdljdp2 & 29.24 & 29.23 & 0.0 \\
\hline 039.wave5 & 21.79 & 22.65 & 4.0 \\
\hline 047.tomcatv & 4.02 & 4.37 & 8.7 \\
\hline 048.ora & 30.95 & 38.35 & 23.9 \\
\hline 052.alvinn & 9.94 & 10.04 & 1.0 \\
\hline 056.ear & 44.48 & 45.18 & 1.5 \\
\hline 077.mdljsp2 & 29.24 & 34.71 & 18.7 \\
\hline 078.swm256 & 36.60 & 35.90 & -1.9 \\
\hline 089.su2cor & 25.15 & 25.77 & 2.5 \\
\hline 090.hydro2d & 28.75 & 28.77 & 0. \\
\hline 093.nasa7 & 27.94 & 28.04 & 0.4 \\
\hline 094.fpppp & 33.62 & 34.64 & 3.0 \\
\hline
\end{tabular}


very flexible framework for implementing and experimenting with different global policies.

In addition to critical path length and latency information (commonly represented as critical path heuristics and delay heuristics in scheduling techniques), the cost model used in this paper also incorporates global register pressure. One effect is that AOM becomes more aggressive in inner loops where more registers are available, and more conservative when moving operations across a region. Our cost model also discourages code expansion and unprofitable code motion, without arbitrarily forbidding duplication. As a result, performance degradation due to AOM is rare.

AOM achieves a more global perspective by considering all movable operations in a $\mathrm{BB}$ pair for profitability in a single unified calculation. By contrast, considering one candidate at a time makes each decision inherently incremental and limited in scope. By looking beyond a single operation, we are able to identify profitable code motion without using complex scheduling heuristics.

Acknowledgments: We would like to thank Fred Chow, Mark Streich, and the referees for their valuable comments and identifying additional references relevant to this work.

A patent application has been filed by Silicon Graphics Computer Systems on the system and method described in this paper.

\section{Bibliography}

[BeRo91] Bernstein, D., and Rodeh, M., Global Instruction Scheduling for Superscalar Machines, Proceedings of the SIGPLAN ' 91 Conference on Programming Language Design and Implementation, pp. 241-255, 1991.

[BaLa93] Ball, T., and Larus, J.R., Branch Predication For Free, Proceedings of the SIGPLAN

'93 Conference on Programming Language Design and Implementation, pp. 300-313, 1993.

[Char+81] Charlesworth, A.E. An Approach to Scientific Array Processing: The Architectural Design of the AP-120B/FPS-164. IEEE Computer 14 (9), pp. 18-27, 1981.

[DeTo93] Dehnert, J.C., and Towle, R.A., Compiling for the Cydra 5, The Journal of Supercomputing 7 (1/2), pp. 181-227, 1993.

[EbNi89] Kemal Ebcioglu and Alexandru Nicolau, A Global Resource-Constrained Parallelization Technique. Proceedings of the 3-rd International Conference on Supercomputing, pp. 154-163, 1989.

[EdKa72] Edmonds, J., and Karp, R.M., Theoretical Improvements in Algorithmic Efficiency for Network Flow Problems, J. Assoc. Computer Machinery 19, pp. 248-264, 1972.

[Ellis86] Ellis, J., Bulldog: A Compiler for VLIW Architectures. MIT Press, Cambridge, Massachusetts, 1986.

[FoFu62] Ford, L.R., and Fulkerson, D.R., Flows in Networks. Princeton University Press, Princeton, New Jersey, 1962.

[Fisher81] Fisher, J.A., Trace Scheduling: A Technique for Global Microcode Compaction. IEEE Transactions on Computers C-30 (7), pp. 478-490, 1981.

[HMCC+93] Hwu, W.W., Mahlke, Chen, Chang, Warter, Bringmann, Ouellete, Hank, Kiyohara, Haab, Holm, and Lavery, The Superblock: An Effective Technique for VLIW and Superscalar Compilation, The Journal of Supercomputing 7 (1/2), pp. 182-229, May 1993.

[Hsu94] Peter Yan-Tek Hsu, Design the TFP Microprocessor, IEEE MICRO, April 1994, pp. $23-$ 33.

[Jain91] Suneel Jain, Circular Scheduling: A New Technique to Perform Software Pipelining, Proceedings of the SIGPLAN '91 Conference on Programming Language Design and Implementation, pp. 219-228, 1991.

[LFKL+93] Lowney, P.G., Freudenberger, S.M., Karzes, T.J., Lichtenstein, W.D., Nix, R.P., O'Donnell, J.J., and Ruttenberg, J.C., The Multiflow Trace Scheduling Compiler, The Journal of Supercomputing 7 (1/2), pp. 51-142, May 1993. 
[Lo88] Virginia Mary Lo, Heuristic Algorithms for Task Assignment in Distributed Systems, IEEE Trans. on Computers, vol. 37, no. 11, pp. 1384-1397, Nov 1988.

[NaEb93] Toshio Nakatani and Kemal Ebcioglu, Making Compaction-Based Parallelization Affordable, IEEE Trans. on Parallel and Dist. Syst. 4(9), pp. 1014-1029, 1993.

[Nicolau86] Alexandru Nicolau, A Fine-Grain Parallelizing Compiler, Tech. Report No. 86792, Cornell University, 1986.

[RaFi93] Rau, B.R., and Fisher, J.A., Instruction-Level Parallel Processing: History, Overview, and Perspective, The Journal of Supercomputing 7 (1/2), pp. 9-50.

[RaG181] Rau, B.R., and Glaeser, C.D., Some Scheduling Techniques and an Easily Schedulable Horizontal Architecture for High-performance Scientific Computing, Proceedings - MICRO14, October 1981, pp. 183-198.

[RGSL96] Ruttenberg, J., Gao, G., Stoutchinin, A., and Lichtenstein, W., Software Pipelining Showdown: Optimal vs. Heuristic Methods in a Production Compiler, to appear in Proceedings of the SIGPLAN '96 Conference on Programming Language Design and Implementation, May 1996.

[Stone77] Harold S. Stone, Multiprocessor Scheduling with the Aid of Network Flow Algorithms, IEEE Trans. on Software Engineering, vol. SE-3, no. 1, Jan 1977.

[Touzeau84] Touzeau, R.F., A Fortran Compiler for the FPS-164 Scientific Computer. In Conference Proceedings - SIGPLAN '84 Symposium on Compiler Construction (Montreal, Canada, June 20), pp. 48-57, 1984.

\section{Appendix}

The following example demonstrates how the movement set is determined for an equivalent code motion. Figure 2 shows the dependency graph of $B B_{s}$ annotated with estart $-E_{t}$ and $l$ start $E_{t}$ information. The shaded nodes are the non-movable operations; others are movable. Negative values indicate that the operation can be moved to $B B_{t}$ without increasing the target path length, while positive values indicate otherwise. The distance $(D)$ between $B B_{s}$ and $B B_{t}$ is assumed to be 10. Figure 3 shows the flow network transformed from the dependency graph. The flow network contains the source node, sink node, and the nodes corresponding to movable operations. Each operation-arc is annotated with a 3-tuple $\left(C_{\text {path }}, C_{\text {reg }}, C_{\text {latency }}\right)$, as defined by the equations in Section 3.3. Distance $(D)$ is 10 and the maximum latency in this movable set is 3 . Therefore the subsumable latency is 3 .

Consider operation 4: "ld $\mathrm{t} 4$, (g2)". The operation's (estart- $E_{t}$, lstart-E $)$ is $(-1,-1)$, and its latency is 3 . Its source-arc is annotated with $(1,0,3)$ and its operation-arc with $(0,1,0)$.

For the source-arc $(0,4)$ :

$$
\begin{aligned}
& \text { estart }\left(\operatorname{arc}_{(0,4)}\right)=\operatorname{estart}\left(\mathrm{op}_{4}\right)-E_{t}=-1 . \\
& \text { lfinish }\left(\operatorname{arc}_{(0,4)}\right)=l \operatorname{start}\left(\mathrm{op}_{4}\right)-E_{t}=-1 . \\
& C_{\text {path }}=- \text { lfinish }\left(\operatorname{arc}_{(0,4)}\right)=1 . \\
& C_{\text {reg }}=0 \text { since its operand "g2" is live-in. } \\
& C_{\text {latency }}=3 \text { (i.e., subsumable latency). }
\end{aligned}
$$

For the operation-arc $\left(4,4^{\prime}\right)$ :

$$
\begin{aligned}
& \text { estart }\left(\operatorname{arc}_{\left(4,4^{\prime}\right)}\right)=\operatorname{estart}\left(\mathrm{op}_{4}\right)-E_{t}=-1 . \\
& \text { lfinish }\left(\operatorname{arc}_{\left(4,4^{\prime}\right)}\right)=l \operatorname{start}\left(\mathrm{op}_{4}\right)+\text { latency }\left(\mathrm{op}_{4}\right)-E_{t}=2 . \\
& C_{\text {path }}=0 . \\
& C_{\text {reg }}=1 \text { because it defines “ } \mathrm{t} 4 \text { ". } \\
& C_{\text {latency }}=\min (0,(\min (3,10)-3))=0 .
\end{aligned}
$$

The 3-tuple can then be converted into a flow capacity $\left(C_{\text {total }}\right)$. Suppose we are concerned with critical path only (for example, there are plenty of registers available and the distance between the BBs is zero). Then we have $C_{t o t a l}=1 * C_{p a t h}$. The cut will be along arcs with $C_{p a t h}$ $=0$. Operations 2,3 , and 4 are moved. Now if we are concerned about register pressure, say $W_{\text {reg }}$ $=2$, we have $C_{\text {total }}=1 * C_{\text {path }}+2 * C_{\text {reg. }}$. The cut will be along the source-arcs. Nothing is moved. Finally, if $C_{\text {latency }}$ is considered, we have $C_{\text {total }}=1 * C_{\text {path }}+2 * C_{\text {reg }}+1 * C_{\text {latency. }}$ The cut will be along arcs $(0,2)$ and $\left(4,4^{\prime}\right)$. Operation 4 is moved. 
FIGURE 2. DAG annotated with estart- $E_{t}$ and lstart- $E_{t}$ information.

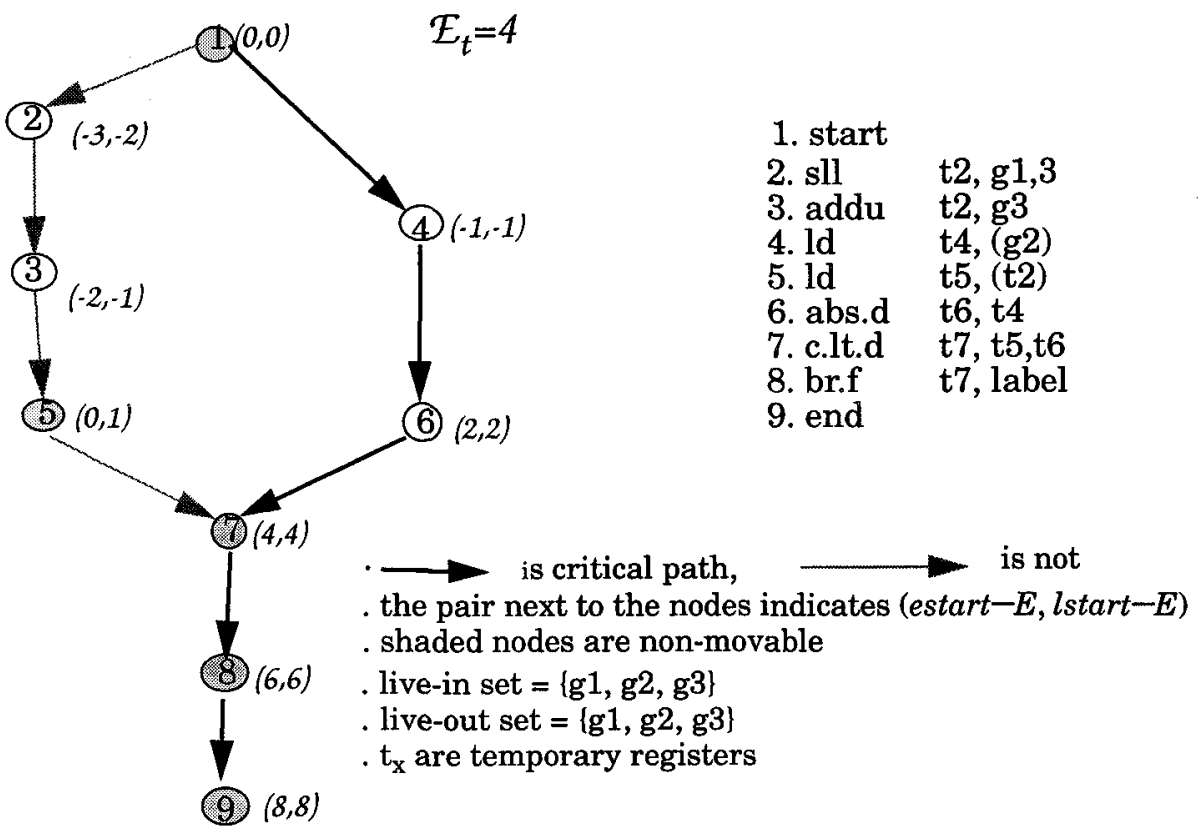

FIGURE 3. Obtaining different cuts with different weight assignment.

the triples indicate capacity assignment as: (critical path, register pressure, latency)

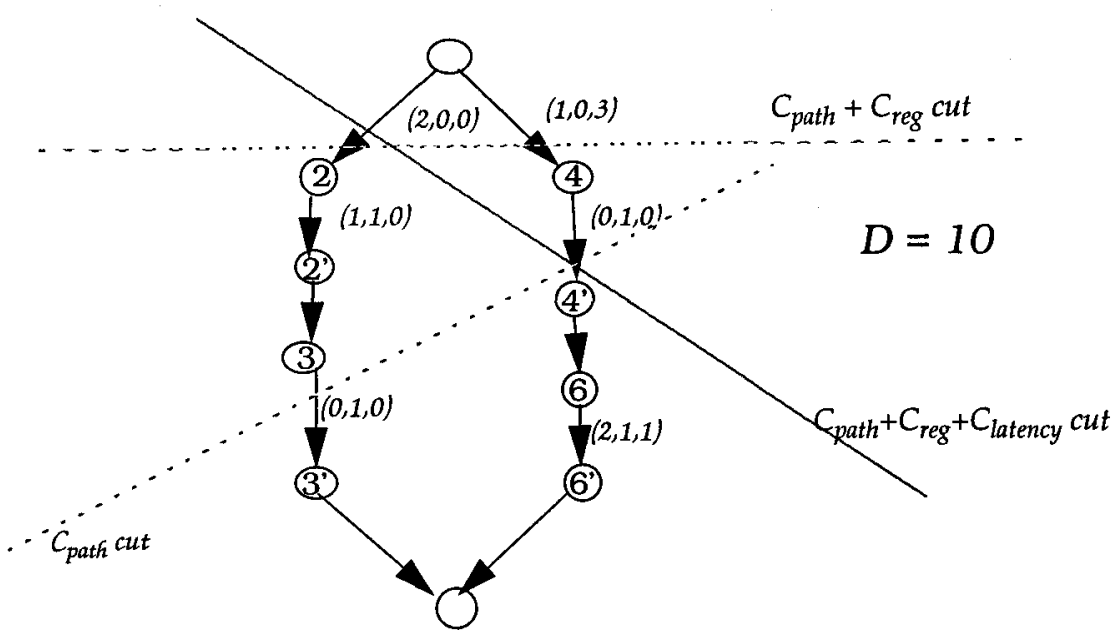

